\title{
Prosthetic Management of Palatally Placed Maxillary Impacted Canine
}

\author{
${ }^{1}$ Vaibhao Ishwar Shambharkar, ${ }^{2}$ Sameera Shaikh, ${ }^{3}$ Shweta Sonare, ${ }^{4}$ Ganesh Bajaj
}

\begin{abstract}
Treatment of asymptomatic impacted maxillary canines in adults is inevitable when primary canine becomes lost through extraction or exfoliation or when the impacted tooth becomes symptomatic. Treatment alternatives include an orthodontic procedure to bring the unerupted tooth to the dental arch or prosthetic replacement of the missing tooth. This is an alternative treatment that involves simultaneous placement of implants into extraction sockets of the teeth for replacement of maxillary canine. This treatment modality avoids the need for conventional preparation of teeth as part of prosthetic reconstruction or prolonged orthodontic treatment aimed at bringing the impacted canine to the dental arch.
\end{abstract}

Keywords: Demineralized freeze-dried bone allograft, Immediate placement of implant, Impacted canine, Implantation with bone augmentation.

How to cite this article: Shambharkar VI, Shaikh S, Sonare S, Bajaj G. Prosthetic Management of Palatally Placed Maxillary Impacted Canine. Int J Prosthodont Restor Dent 2015;5(2): 56-59.

\section{Source of support: Nil}

Conflict of interest: None

\section{INTRODUCTION}

With the exception of third molars, the most frequently impacted teeth in adults are maxillary canines. ${ }^{1}$ Unerupted permanent canines cause relatively few problems for patients, and some of these teeth remain unerupted and asymptomatic for many years. Although a retained primary canine may result in a relatively poor appearance compared with that of a permanent canine; most patients

\footnotetext{
${ }^{1,2}$ Assistant Professor, ${ }^{3}$ Private Practitioner

${ }^{4}$ Associate Professor

${ }^{1,4}$ Department of Prosthetic Dentistry, SDKS Dental College and Hospital, Nagpur, Maharashtra, India

${ }^{2}$ Department of Prosthetic Dentistry, Government Dental College and Hospital, Mumbai, Maharashtra, India

${ }^{3}$ Department of Dentistry, Vaibhao Dental Clinic, Nagpur Maharashtra, India

Corresponding Author: Vaibhao Ishwar Shambharkar Assistant Professor, Department of Prosthetic Dentistry SDKS Dental College and Hospital, Nagpur, Maharashtra India, Phone: 9028404514, e-mail: vaibhao.shambharkar@ gmail.com
}

do not seek treatment for a retained primary canine. The reasons why adults have impacted maxillary canines vary. In some cases, the general dentist or orthodontist may not have emphasized the importance of treating the impacted permanent tooth when the patient was a child. Or perhaps the parents had an inadequate level of awareness about dental health issues. Some impacted canines simply may never have been diagnosed. Another possibility is that the parent-and later the patientchose to delay treatment until an esthetic or functional need arose or the impacted tooth became symptomatic. Before initiating orthodontic treatment to resolve the impaction, the dentist must inform the adult patient that teeth that have been impacted for many years sometimes undergo pathological changes that might prevent their eruption. ${ }^{2}$ Dentist must carefully inform the patient of the significant possibility of failure when attempting to bring an impacted tooth into the arch. Moving the canine to the dental arch takes considerably longer time in an adult than in a child. Therefore, the patient must be part of the decision-making process and be fully informed of the potential problems related to treatment replacing missing teeth. Dental implants is a common treatment procedure to manage such situations. ${ }^{3,4}$ Several authors have reported success rates of more than $90 \%$ for extraction sites. ${ }^{5-8}$ In many cases, the surgeon faces difficulty when immediately placing implants because usually there is a gap between the occlusal section of the implant and the surrounding socket walls. But in this particular case there was sufficient amount of bone up to 7 to $8 \mathrm{~mm}$ was present surrounding the socket wall. So there was no need to place the graft material. Primary stability was achieved by 7 to $8 \mathrm{~mm}$ of bone present surrounding the implant. ${ }^{9-11}$ This problem can also be resolved by using a variety of grafting mate implants placed into fresh materials with a barrier membrane, which encourages bone to fill the empty space. ${ }^{12-14}$

\section{CASE REPORT}

\section{Diagnosis}

A 24 years old male patient visited to the Department of Prosthodontics, Government Dental College and Hospital Nagpur, for replacement of his missing maxillary left 
canine. The Intraoral periapical radiograph (IOPA) was taken as a routine examination protocol. The IOPA examination revealed horizontally placed permanent left canine in periapical region (from left first premolar to right central incisor) with crown directed anteriorly (Fig. 1). It was classified the patient's status as Angle class I malocclusion, with unilateral palatally impacted canine. The patient's dental history revealed that, he visited a private practitioner 1 year back, due to the horizontal fracture of the left primary canine, which was later extracted. The patient in this case did not seek treatment earlier because of the esthetically acceptable primary maxillary canine and asymptomatic impacted permanent canine (Fig. 2). Both orthodontic and prosthetic options were suggested to replace the clinically missing permanent canine. Considering the risk that the impacted teeth might fail to erupt and the need for a protracted treatment period if eruption was successful, the patient chose a prosthetic approach. Two treatment modalities were possible: an anterior fixed conventional bridge or a semibonded (Maryland-type) bridge with or without extraction of the impacted canines; surgical removal of the impacted teeth combined with placement of implants. The patient chose the second option.

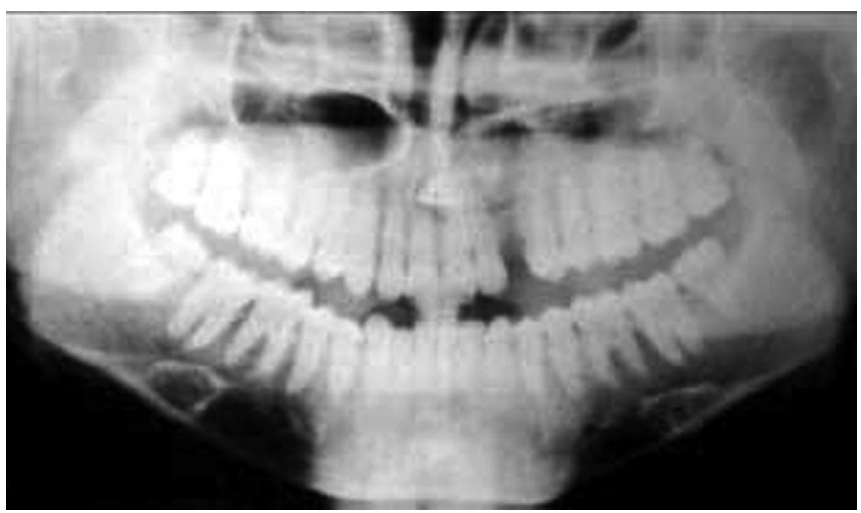

Fig. 1: Horizontally placed permanent left canine

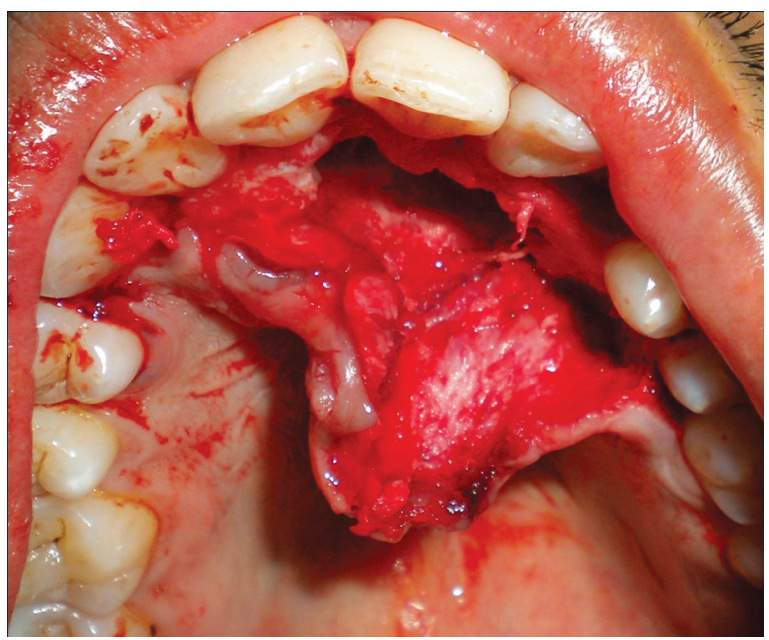

Fig. 3: Palatal flap was reflected to expose the impacted canines

\section{Implant Placement}

On clinical examination sufficient mesiodistal space was available so there was no need to orthodontically reopen the mesiodistal width of the canine area. After local anesthetic was administered, the palatal flap was reflected to expose the impacted canines and the alveolar crest. Conservative treatment approach was applied while extracting the canine (Fig. 3). Maxillary impacted left canine was extracted and immediate implant placement was done. Hydroxyapatitecoated implants (hi-tech, Israel) that were 3.75 millimeters in diameter and $13 \mathrm{~mm}$ in length were inserted (Fig. 4). After 6 months healing period IOPA was taken to ensure the bone deposition in the extraction socket of the horizontally impacted canine. A panoramic radiograph and IOPA radiographs were obtained 6 months after surgery showed complete osseous fill of the defect. After 6 months when the implants were reexposed for preparation of the final restoration, no sign of bone loss was evident and the implants were clinically stable. The implants were uncovered, cover screws (hi-tech, Israel) were removed and healing caps (hi-tech, Israel) were placed for several days (Fig. 5). One month after second-stage surgery, the healing caps were removed,

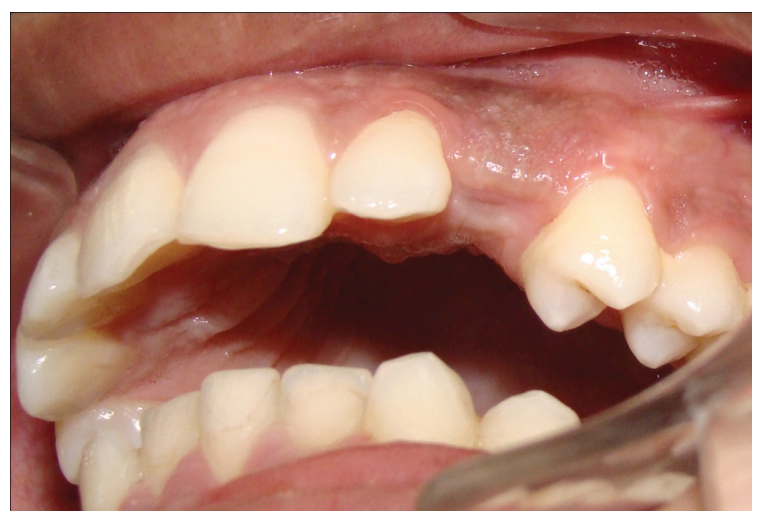

Fig. 2: Preoperative photograph

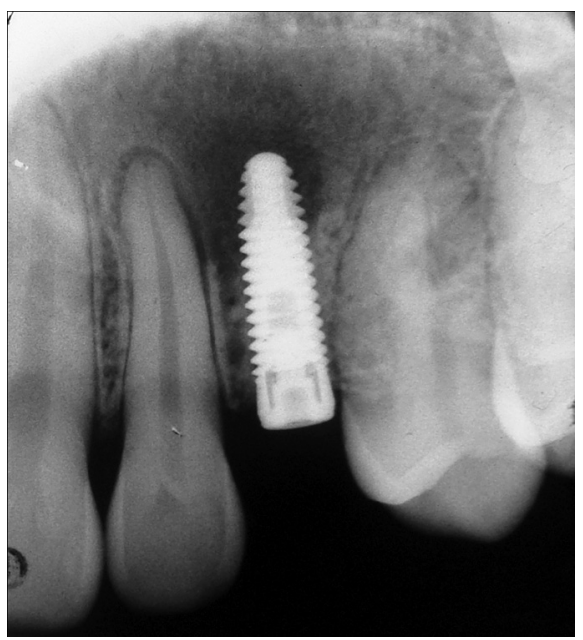

Fig. 4: Implant placement 


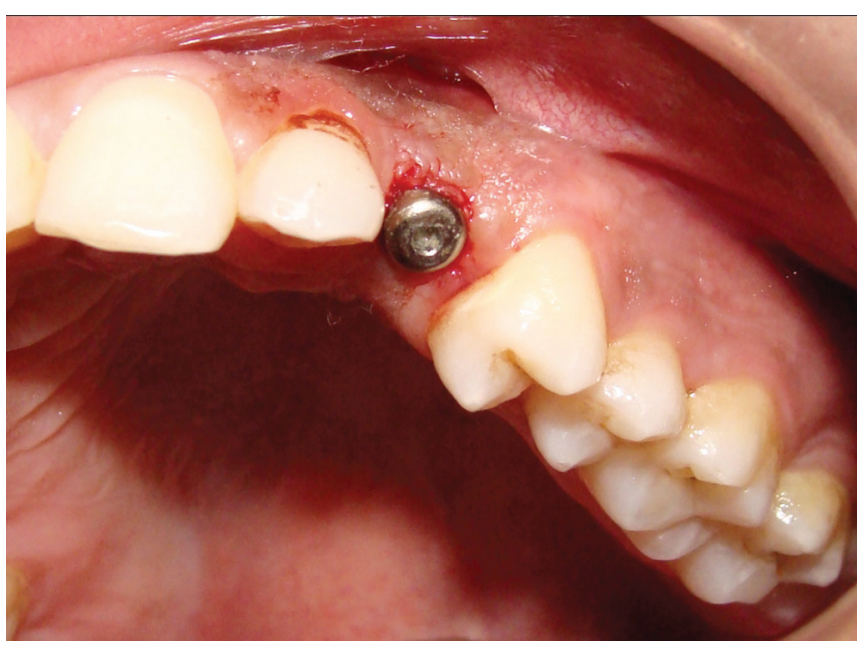

Fig. 5: Placement of healing cap

and definitive impressions were made (Fig. 6). The abutment positions relative to the adjacent teeth were evaluated, and clearance in maximum intercuspation and excursive movements was ensured. After corrections were made to the implant abutments they were evaluated intraorally for fit. Periapical radiographs were made to ascertain the implant abutment fit to the implant. Metal ceramic complete crowns were then fabricated. The crowns were subsequently evaluated for marginal fit, proximal contacts, esthetics, and occlusal contacts. A group function occlusal scheme was selected to involve contacts of the incisors in lateral movements rather than using a canine-guided articulation. The patient approved the esthetic result at the trial insertion appointment. The patient was very satisfied with the final result. One year later, clinical and radiographic examinations revealed satisfactory esthetics, function and marginal bone integrity (Fig. 7). We have presented a case report that illustrates a unique treatment modality for impacted canines in an adult. The simultaneous placement of an implant into the extraction site of a palatally impacted canine shortened the treatment period.

\section{DISCUSSION}

This is a case report that illustrates a unique treatment modality for impacted canine in an adult. The simultaneous

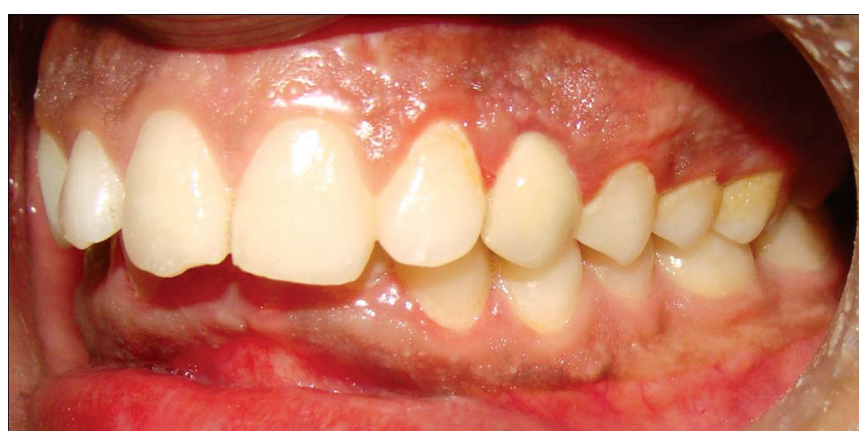

Fig. 7: Final prosthesis

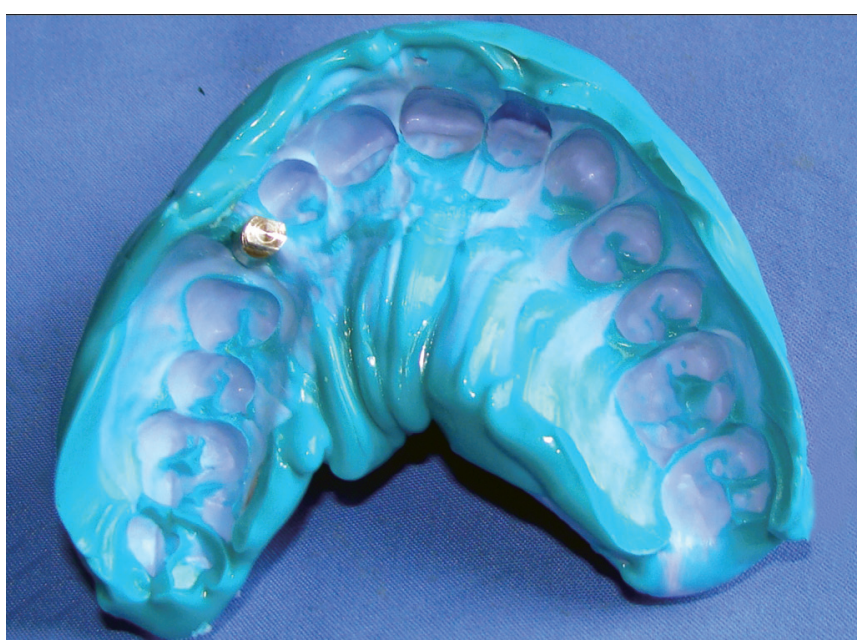

Fig. 6: Definitive impression

placement of an implant into the extraction site of a palatally impacted canine shortened the treatment period. We need to emphasize, however, that a prerequisite for immediate placement of an implant into an extraction site is an adequate mesiodistal arch space for subsequent placement of a crown. In this particular case along with sufficient mesiodistal space, 7 to $8 \mathrm{~mm}$ of bone was present surrounding the implant. This bone was utilized for primary support of the implant. Primary stability was achieved and the apical portion of the implant was held in the cavity which is formed after removing of the canine. A radiographic examination revealed the changes in bone density before the implant placement and after the implant placement. A true lateral radiograph of the incisor region assists in locating the impacted tooth that are lying deeply in the palate and enables the practitioner to decide whether a buccal rather than a palatal approach should be used to remove them. In this case swelling was present on the palatal aspect of the anterior region of the maxilla. So palatal approach was used here to remove impacted canine because it gives proper access to the site. Treatment depends on the type and position of the impacted tooth and on its effect or potential effect on adjacent teeth. The management of a impacted tooth should form part of a comprehensive treatment plan and should not be considered in isolation.

\section{REFERENCES}

1. Becker A. The orthodontic treatment of impacted teeth. London: Martin Dunitz; 1998:179-198.

2. Azaz B, Shteyer A. Resorption of the crown in impacted maxillary canine: a clinical, radiographic and histologic study. Int J Oral Surg 1978;7(3):167-171.

3. Adell R, Lekholm U, Rockler B, Branemark PI. A 15-year study of osseointegrated implants in the treatment of the edentulous jaw. Int J Oral Surg 1981;10(6):387-416.

4. Van Steenberghe D, Lekholm U, Bolender C, et al. Applicability of osseointegrated oral implants in the rehabilitation of 
partial edentulism: a prospective multicenter study on 558 fixtures. Int J Oral Maxillofac Implants 1990;5(3):272-281.

5. Ashman A. An immediate tooth root replacement: an implant cylinder and synthetic bone combination. J Oral Implantol 1990;16(1):28-38.

6. Yukna RA. Clinical comparison of hydroxyapatite-coated titanium dental implants placed in fresh extraction sockets and healed extraction sites. J Periodontol 1991;62(7):468-472.

7. Gelb DA. Immediate implant surgery: three-year retrospective evaluation of 50 consecutive cases. Int J Oral Maxillofac Implants 1993;8(4):388-399.

8. Schwartz-Arad D, Chaushu G. The ways and wherefores of immediate placement of implants into fresh extraction sites: a literature review. J Periodontol 1997;68(10):915-923.

9. Becker W, Dahlin C, Becker BE, et al. The use of e-PTFE barrier membranes for bone promotion around titanium implants placed into extraction sockets: a prospective multicenter study. Int J Oral Maxillofac Implants 1994;9(1):32-40.
10. Gher ME, Quintero G, Assad D, Monaco E, Richardson AE. Bone grafting and guided bone regeneration for immediate implants in humans. J Periodontol 1994;65(9):881-891.

11. Bragger $\mathrm{U}, \mathrm{Hammerle} \mathrm{CH}$, Lang NP. Immediate transmucosal implants using the principle of guided tissue regeneration. II. A cross-sectional study comparing the clinical outcome 1 year after immediate to standard implant placement. Clin Oral Implants Res 1996;7(3):268-276.

12. Augthun M, Yildirim M, Spiekermann H, Biesterfeld S. Healing of bone defects in combination with immediate implants using the membrane technique. Int J Oral Maxillofac Implants 1995;10(4):421-428.

13. Block MS, Kent JN. Placement of endosseous implants into tooth extraction sites. J Oral Maxillofac Surg 1991;49(12): 1269-1276.

14. Kent JN, Block MS. Simultaneous maxillary sinus floor bone grafting and placement of hydroxylapatite-coated implants. J Oral Maxillofac Surg 1989;47(3):238-242. 